\title{
Magnetic Characterization of Iron Oxide Nanoparticles for Biomedical Applications
}

\author{
Lorena Maldonado-Camargo, Mythreyi Unni, and Carlos Rinaldi
}

\begin{abstract}
Iron oxide nanoparticles are of interest in a wide range of biomedical applications due to their response to applied magnetic fields and their unique magnetic properties. Magnetization measurements in constant and time-varying magnetic field are often carried out to quantify key properties of iron oxide nanoparticles. This chapter describes the importance of thorough magnetic characterization of iron oxide nanoparticles intended for use in biomedical applications. A basic introduction to relevant magnetic properties of iron oxide nanoparticles is given, followed by protocols and conditions used for measurement of magnetic properties, along with examples of data obtained from each measurement, and methods of data analysis.
\end{abstract}

\section{Keywords}

Magnetic nanoparticles; Anisotropy constant; Blocking temperature; Magnetic relaxation; Saturation magnetization; Remanent magnetization; Coercivity

\section{Introduction}

Iron oxide magnetic nanoparticles have been widely used due to the capability of manipulating particle motion, causing energy dissipation, or providing imaging contrast in the presence of an external magnetic field [1,2]. Their use in biomedical applications such as cancer therapy [3, 4], magnetically triggered drug release [5, 6], magnetofection [7, 8], magnetic resonance imaging $[9,10]$, and magnetic particle imaging $[11,12]$ has been widely researched. Examples of important magnetic properties of nanoparticles include the saturation magnetization, remanence and coercivity, magnetic diameter, magnetocrystalline anisotropy constant, mechanism of magnetic relaxation, and blocking temperature, all of which can be material specific and may be influenced by the method of synthesizing and coating the nanoparticles and by the method of sample preparation for magnetic measurements. Accurately quantifying these properties is vital to enable reproducibility in research and to achieve the maximum potential of iron oxide nanoparticles in specific applications. Unfortunately, many publications describing the preparation and use of magnetic nanoparticles lack even basic magnetic characterization, making it difficult to evaluate and compare the work. The methods described in this chapter present detailed procedures to determine the magnetic properties of iron oxide nanoparticles that most significantly impact their biomedical applications. 
The magnetic response of iron oxide nanoparticles to an external field depends mainly on the degree of magnetic ordering and on the temperature of the sample. The magnetic moment per unit volume of particle, i.e., the magnetization, may be defined depending on the spin or the orbital energy possessed by the dipole. Particles with large crystallite sizes have dipoles arranged in multiple domains separated by a domain wall so as to maintain the lowest energy state. There exists a critical size (typically less than $100 \mathrm{~nm}$ ) below which it is energetically unfavorable for domain walls to form, resulting in single domain nanoparticles [13]. As predicted by Louis Néel, nanoparticles in the single domain regime no longer exhibit hysteresis behavior in an applied magnetic field, a condition that is referred to as superparamagnetism [14]. The most commonly used magnetic nanoparticles are ferrites $\mathrm{MFe}_{2} \mathrm{O}_{4}$ that exhibit a spinel or inverse spinel structure. The distribution of the metal ions relative to the oxygen ions in the crystal lattice results in the formation of dipoles and determines the overall magnetization of the material. A measure of this ordering and the strength of the dipoles in single domain particles may be obtained from the magnetic diameter obtained from fitting the Langevin equation (see Subheading 3.5) to an experimental equilibrium magnetization curve $[15,16]$.

In the presence of a magnetic field, magnetic spins tend to align in the direction of the field, resulting in an induced magnetization [17]. The maximum induced magnetization is termed the saturation magnetization of the sample. This induced magnetization may remain even after the field is removed, in which case it is referred to as the remanent magnetization. In those cases, the coercive field corresponds to the magnetic field required to revert the magnetization to zero. Nanoparticles with ferro- and ferri-magnetic behavior often exhibit hysteresis. In nanoparticles that exhibit superparamagnetism, remanence and coercive field becomes negligible.

Nanoparticles show a certain preference for the direction along which their magnetic dipole tends to align, referred to as magnetic anisotropy, which can arise due to the shape and inherent crystalline structure of the nanoparticles. This anisotropy can be intrinsic to the material such as magnetocrystalline, shape, and exchange anisotropy, or induced by an external process. Magnetocrystalline anisotropy refers to the tendency of the magnetization to align along a preferred crystallographic direction. In contrast, polycrystalline samples with no preferred crystal orientation tend to magnetize along a long axis, in what is known as shape anisotropy. Finally, exchange anisotropy arises from interaction between antiferromagnetic and ferromagnetic materials $[13,17]$. The rate at which the magnetic dipole within a particle will align in a given direction of applied magnetic field is influenced by the temperature of the system (thermal energy) and the magnitude of the magnetic anisotropy energy barrier between easy axes for magnetization.

The magnitude of the magnetocrystalline anisotropy can be determined by temperaturedependent magnetization measurements such as zero-field-cooled (ZFC) and Dynamic Magnetic Susceptibility (DMS) curves in samples wherein nanoparticles' physical rotation is suppressed, either by freezing the sample or dispersing it in a solid matrix (see Subheadings 3.7 and 3.8). 
There are two mechanisms by which the magnetization of a colloidal suspension of nanoparticles responds after the removal of an external applied field [18-20]. In the first mechanism, the relaxation of the magnetic dipole occurs by physical particle rotation in the liquid. The corresponding characteristic rotational diffusion time $\tau_{B}$, referred to as the Brownian relaxation time, is given by

$$
\tau_{\mathrm{B}}=\frac{3 V_{\mathrm{h}} \eta}{k T}
$$

where $\eta$ is the viscosity of the carrier liquid, $V_{\mathrm{h}}$ is the hydrodynamic volume of the particle, $k$ is the Boltzmann constant, and $T$ is the temperature. In the second mechanism, the magnetic dipole rotates within the particle. The corresponding characteristic time $\tau_{\mathrm{N}}$ for dipole rotation is termed the Néel relaxation time and is given by

$$
\tau_{\mathrm{N}}=\tau_{0} \exp \left(\frac{K V_{\mathrm{m}}}{k T}\right)
$$

where $\tau_{0}$ is a characteristic time with an approximate value of $10^{-9} \mathrm{~s}, V_{\mathrm{m}}$ is the magnetic core volume, and $K$ is the anisotropy constant. In a colloidal suspension of nanoparticles, both relaxation mechanisms are present but the faster mechanism dominates. The Brownian relaxation time is proportional to the viscosity of the carrier liquid and the particle hydrodynamic diameter, whereas the Néel mechanism is solely related to the volume of the magnetic core and the anisotropy constant of the material. Dynamic Magnetic Susceptibility (DMS) measurements (see Subheadings 3.9) can be used to obtain information of the magnetic relaxation properties of nanoparticles in suspension, including measurements of their characteristic magnetic relaxation time.

Here, we describe in detail how superconducting quantum interference device (SQUID) magnetometers and dynamic magnetic susceptometers can be applied to quantify magnetic properties of iron oxide nanoparticles in a liquid or solid matrix. Sample preparation methods and data analysis are also explained. We remark that determining properties of nanomaterials is often difficult on the basis of a single technique. The methods described in this chapter should provide a fairly complete assessment of the magnetic properties of iron oxide nanoparticles that most significantly impact their biomedical applications including low and high field magnetization curves, Zero-Field-Cooled/Field-Cooled (ZFC/FC) magnetization curves, and Dynamic Magnetic Susceptibility (DMS) measurements. However, there are other magnetic measurements, such as isothermal reversibility measurements, First Order Reversal Curves (FORC), and Verwey transition analysis that we have left out of this chapter [21-25]. The reader may adapt this protocol to characterize their specific nanoparticle of interest by taking into consideration the compatibility of the particle surface coating with the polymer or solvent selected. 


\section{Materials}

\subsection{Immobilization of Hydrophobic Nanoparticles in a Solid Matrix (See Note 1)}

1. Magnetic nanoparticles synthesized or obtained commercially and coated with organic molecules such as oleic acid, hydrophobic polymers, and oleylamine.

2. Styrene ReagentPlus ${ }^{\circledR}$, containing 4-tert-butylcatechol as a stabilizer, $\searrow 99 \%$. Store at $4{ }^{\circ} \mathrm{C}$.

3. Divinylbenzene technical grade, $80 \%$ (DVB). Store at $4{ }^{\circ} \mathrm{C}$.

4. $2,2^{\prime}$-Azobis (2-methylpropionitrile) (AIBN). Store at $4{ }^{\circ} \mathrm{C}$.

5. $3 \mathrm{~mL}$ glass vial.

6. $\quad 0.7 \mathrm{~mL}$ glass test tubes with screw cap (6 mm OD, $50 \mathrm{~mm}$ length).

7. Ultrasonicator probe fitted with a tapered microtip ( $3 / 6$ in).

8. Oil bath.

\subsection{Immobilization of Hydrophilic Nanoparticles in a Solid Matrix (See Note 2)}

1. Magnetic nanoparticles synthesized or obtained commercially and coated with hydrophilic molecules, such as hydrophilic polymers, amines, carboxylic groups or peptized.

2. Tetraethylene glycol dimethacrylate (TEGDMA), technical grade, $\geq 90 \%$. Store at $4{ }^{\circ} \mathrm{C}$.

3. $2,2^{\prime}$-Azobis(2-methylpropionitrile) (AIBN). Store at $4{ }^{\circ} \mathrm{C}$.

4. $3 \mathrm{~mL}$ glass vial.

5. $\quad 0.7 \mathrm{~mL}$ glass test tubes with screw cap (6 mm OD, $50 \mathrm{~mm}$ length).

6. Ultrasonicator probe fitted with a tapered microtip (3/6 in).

7. Oil bath.

\subsection{Nanoparticle Suspensions}

1. Magnetic nanoparticles: Iron oxide, cobalt ferrite, manganese ferrite, etc., synthesized or obtained commercially. This protocol uses iron oxide nanoparticles.

2. Toluene, hexane, chloroform, 1-octadecene, tetrahydrofuran, or any organic solvent suitable to suspend particles coated with organic molecules, such as oleic acid or oleylamine. This protocol uses iron oxide nanoparticles coated with oleic acid suspended in 1-octadecene.

\footnotetext{
1. Any compatible solid matrix can be used to restrict nanoparticle rotation, for example paraffin wax, docosane, or higher hydrocarbons. A solid polymer matrix of polystyrene-divinyl benzene is used for this protocol due to its high thermal resistance, allowing measurements at up to $400 \mathrm{~K}$.

2. Any compatible solid matrix can be used to restrict nanoparticle rotation, for example silica, agar, etc. A solid polymer matrix of TEGDMA is used for this protocol due to its higher thermal resistance, allowing measurements at up to $400 \mathrm{~K}$.
} 
3. Water or other suitable polar solvents to suspend particles coated with hydrophilic molecules, such as hydrophilic polymer, amines, carboxylic groups, or peptized. This protocol uses water.

4. Filters: Nylon filters for filtration of aqueous solutions. Polytetrafluoroethylene (PTFE) filters for filtration of organic solvents. Polyvinylidene fluoride (PVDF) filters for filtration of nonaggressive aqueous and mild organic solutions (see Note 3).

5. Mechanical ultrasonic bath.

\subsection{Equipment}

1. Dynamic magnetic susceptometers operating at low amplitude fields $\sim 0.5 \mathrm{mT}$ with an ideal excitation frequency ranging from 1 to $100 \mathrm{kHz}$ (such as Acreo DynoMag).

2. Vibrating sample magnetometer (VSM) (such as 7400-S from Lake shore Cryotronics, or VSM from Quantum Design).

3. Superconducting Quantum Interference Device (SQUID) magnetometer (such as MPMS3 from Quantum Design or S700X from Cryogenic Ltd) (see Note 4).

\section{Methods: Sample Preparation}

As seen in the introduction, magnetic nanoparticles may relax by either of two mechanisms; Néel and Brownian relaxation. Distinguishing between these two mechanisms of magnetic relaxation is critical in many biomedical applications, as physical particle rotation may be significantly impaired once nanoparticles accumulate in tissues or inside cells. Measuring magnetic properties for nanoparticles fixed in a solid matrix allows one to abrogate the effects of Brownian relaxation in the magnetic response of the nanoparticles during a measurement, whereas when the nanoparticles are in suspension both mechanisms contribute to the response. As such, characterization of samples in liquid and solid matrices can provide useful insights into their relaxation-dependent properties.

\subsection{Immobilization of Hydrophobic Nanoparticles in a Solid Matrix}

1. Prepare a stock solution of the monomers and initiator by mixing $3 \mathrm{~mL}$ of styrene, $450 \mu \mathrm{L}$ of DVB, and $10 \mathrm{mg}$ of AIBN in a $10 \mathrm{~mL}$ glass vial (see Note 5).

\footnotetext{
3. The membrane used should be selected based on its compatibility with the nanoparticle surface chemistry. The most commonly used filters are nylon filters for hydrophilic solutions, i.e., nanoparticles suspended in water-based solvents, and PTFE filters for hydrophobic solutions, i.e., nanoparticles suspended in organic solvents. The size of the filter is selected based on the particle size. Typically, $0.2 \mu \mathrm{m}$ filters are used for nanoparticles with a size range between 10 and $100 \mathrm{~nm}$. After filtration, the solution may look diluted. This is because some particles and/or particle aggregates are retained by the filter. One should quantify the magnetic concentration of the solution after filtration using a suitable method (see Note 6). Some particles tend to aggregate or become unstable in solution. We recommend not to filter such solutions since the particles are trapped by the filter.

4.SQUID magnetometers are designed to be extremely sensitive $\left(10^{-8} \mathrm{emu}\right)$, whereas the most commonly used vibrating sample magnetometer with an inductive pick up coil are less sensitive $\left(10^{-6} \mathrm{emu}\right)$ but can make faster measurements.

5. Work with all chemicals inside a fume hood. The stock solutions of monomers and initiator must have a 6.66:1 volume ratio of styrene to DVB, and $3.3 \mathrm{mg}$ of AIBN per $1 \mathrm{~mL}$ of styrene. We scale up the solution volume to $3 \mathrm{~mL}$ to accurately weigh the AIBN. The monomer/initiator solution can be stored at $4{ }^{\circ} \mathrm{C}$ for 1 month.
} 
2. Weigh $\sim 10 \mathrm{mg}$ of the magnetic nanoparticle sample into a glass vial. The weight percentage of the magnetic core in the sample must be measured prior to immobilization in a solid matrix, so as to properly estimate the amounts for the various components (see Note 6).

3. Add appropriate amount of the monomer/initiator solution to the nanoparticles in the glass vial to obtain a concentration of magnetic core of $\sim 0.1 \mathrm{wt} \%$ (see Note 7). For example, if the nanoparticle sample (i.e., magnetic core and the ligands on the particles) is $15 \mathrm{wt} \%$ of magnetic core and $85 \mathrm{wt} \%$ ligands on the particle, add $1.5 \mathrm{~g}$ of the monomer/initiator solution $(1.5 \mathrm{~mL}$ assuming density $\sim 1 \mathrm{~g} / \mathrm{mL})$ to $0.01 \mathrm{~g}$ of magnetic nanoparticle sample, such as the final magnetic core concentration in the solution is $0.1 \mathrm{wt} \%$.

4. Shake the solution vigorously. If necessary, use an ultrasonicator probe fitted with a tapered microtip (3/16 in.) to disperse the particles in the polymer.

5. Place $500 \mu \mathrm{L}$ of the solution in glass test tubes with screw-cap (Fig. 1).

6. Place the tube in an oil bath and increase the temperature to $70{ }^{\circ} \mathrm{C}$.

7. Allow the reaction to proceed for $4 \mathrm{~h}$. The monomer should be completely polymerized, forming a solid matrix (Fig. 1).

8. To release the polymer from the tube, tap the sides of the tube. Do this lightly and be careful not to break the sample or the glass tube.

9. Weigh the sample to account for any evaporation losses and recalculate the nanoparticle concentration if needed.

\subsection{Immobilization of Hydrophilic Nanoparticles in a Solid Matrix}

1. Weigh $\sim 10 \mathrm{mg}$ of the magnetic nanoparticle sample into a glass vial. The weight percentage of the magnetic core in the sample must be measured prior to immobilization in a solid matrix, so as to properly estimate the amounts for the various components (see Note 6).

2. Add the appropriate amount of the tetraethylene glycol dimethacrylate (TEGDMA) to obtain a concentration of magnetic core of $\sim 0.1 \mathrm{wt} \%$ ( see Note 7). For example, to embed $0.01 \mathrm{~g}$ of magnetic nanoparticles with a $10 \mathrm{wt} \%$ of magnetic core (i.e., $10 \mathrm{wt} \%$ magnetic core and $90 \mathrm{wt} \%$ polymer coating), add 1.0 $\mathrm{g}$ of the monomer/initiator solution $(1.0 \mathrm{~mL}$ assuming density $\sim 1 \mathrm{~g} / \mathrm{mL})$, such as the final magnetic core concentration in the solution is $0.1 \mathrm{wt} \%$.

\footnotetext{
6. This is the concentration of inorganic magnetic core, i.e., not counting the ligands or polymers on the particle surface. To study magnetic properties, determining the inorganic core content is important. A few commonly used techniques include quantification using UV spectrometric assays [35], inductively coupled plasma mass spectrometry (ICP-MS) [36], electron paramagnetic resonance [37], and thermal gravimetric analysis [38]. Of these, we prefer EPR, ICP-MS, and UV spectrophotometric assays because they can determine the amount of iron in a sample accurately. For UV spectrophotometric and ICP-MS assays care should be taken while digesting samples in concentrated $\mathrm{HNO} 3$ or $\mathrm{HCl}$ during sample preparation.

7. Although the concentration of magnetic core can be increased, we have found that for concentrations of $1 \mathrm{wt} \%$ ( $\mathrm{mg}$ of nanoparticles per mg of polymer) and higher, the calculated value of magnetic properties such as anisotropy constant and the characteristic time are apparent and represent the effective property of the collection of nanoparticles and not an intrinsic property [33].
} 
3. Shake the solution vigorously. If necessary, use an ultrasonicator probe fitted with a tapered microtip (3/16 in.) to disperse the particles in the polymer.

4. Add AIBN to the previous solution to a concentration of $0.04 \mathrm{wt} \%$.

5. Place $500 \mu \mathrm{L}$ of the solution in glass test tubes with screw-cap.

6. Place the tube in an oil bath and increase the temperature to $70{ }^{\circ} \mathrm{C}$.

7. Allow the reaction to proceed for $4 \mathrm{~h}$. The monomer should polymerize, forming a solid matrix.

8. To release the polymer from the tube, tap the sides of the tube. Do this lightly and be careful not to break the sample or the glass tube.

9. Weigh the sample to account for any evaporation losses and recalculate particle concentration if needed.

\subsection{Suspending Nanoparticles in a Liquid Matrix}

1. Weigh $\sim 10 \mathrm{mg}$ of the magnetic nanoparticle sample. The weight percentage of the magnetic core in the sample must be measured prior to suspension in a liquid matrix, so as to properly estimate the amounts for the various components (see Note 6).

2. Add the approximate amount of solvent (organic for hydrophobic nanoparticles, water for hydrophilic nanoparticles) to obtain a concentration of magnetic cores of $\sim 0.1 \mathrm{wt} \%$ (Fig. 1c, see Note 7).

3. Use a sonic bath to suspend the particles in the solvent.

4. Filter the solution using a syringe filter.

\subsection{Methods: Magnetic Measurements and Data Analysis}

For demonstration purposes this chapter describes the use of a solid sample prepared with oleic acid coated iron oxide nanoparticles embedded in a poly(styrene-divinyl benzene) matrix (PSDVB), which inhibits particle rotation, and nanoparticles suspended in 1oactadecene to allow for nanoparticle rotation. The reader may adapt this protocol to characterize their specific nanoparticle of interest by taking into consideration the compatibility of the particle surface with the polymer or solvent selected.

In the following protocol, a Quantum Design MPMS3 Superconducting Quantum Interference Device (SQUID) magnetometer is used in Subheadings 3.5-3.8 to determine the saturation magnetization, remanence and coercivity, magnetic diameter, magnetocrystalline anisotropy constant, and blocking temperature solid samples. An Acreo DynoMag dynamic magnetic susceptometer is used in Subheading 3.9 to estimate the mechanism of magnetic relaxation of the nanoparticles in a liquid matrix. The reader is assumed to be familiar with the equipment and relevant software described associated with these instruments, or the instruments chosen to carry out similar characterization experiments. 


\subsection{Magnetization Vs. Magnetic Field (MH) at Constant Temperature}

The MH curve allows one to verify the superparamagnetic behavior of the nanoparticles and determine their saturation magnetization. Furthermore, a superparamagnetic MH curve can be analyzed by fitting to the Langevin function and a lognormal size distribution to estimate the magnetic diameter of the nanoparticles [15, 16]. Finally, in cases where the nanoparticles are not superparamagnetic, the equilibrium magnetization curve can be used to determine the remanence and coercivity of the nanoparticles at a given temperature. It should be noted that to properly verify superparamagnetic behavior in a sample the nanoparticles should be prevented from rotating. This is because a collection of ferro/ferrimagnetic nanoparticles in liquid suspension will be able to physically rotate to align their magnetic dipoles in the direction of the magnetic field, resulting in an MH curve that lacks remanence and coercivity. This situation is called extrinsic supaerparamagnetism, whereas the case when the nanoparticles are fixed in a matrix and their MH curve lacks remanence and coercivity and follows the sigmoid function (typical S-shape) is called intrinsic superparamagnetism [16]. This is not to say that MH curves should not be obtained for liquid samples, as such measurements can still yield the magnetic diameter of the nanoparticles and can also be used to estimate the volume fraction of nanoparticles in the suspension. In a typical measurement, the magnetization (magnetic moment per unit of volume) of a sample is measured as a function of the applied magnetic field at constant temperature. The magnetic diameter distribution, saturation magnetization, coercive field and remanent magnetization can all be determined from $\mathrm{MH}$ curves.

1. Fix the magnetic nanoparticles in a solid matrix as explained in Subheadings 3.1 and 3.2. In this protocol we use iron oxide nanoparticles in a PSDVB matrix. The reader may adapt this protocol to use a polymer or solid matrix compatible with their nanoparticle system (see Notes 1 and 2).

2. Set the desired sample temperature using the equipment software (see Note 8). We use a Superconducting Quantum Interference Device (SQUID) magnetometer MPMS3 from Quantum Design. The reader is assumed to be familiar with the equipment and relevant software described in Subheading 2.4.

3. Measure the magnetization $M(H)$ of the sample with increasing magnetic field. Use of an applied magnetic field range of 7 to $-7 \mathrm{~T}$ with a field ramp of 200 Oe/min is highly recommended (see Note 9). In Fig. 2a, the magnetization curve of iron nanoparticles is measured at different saturation fields.

4. The magnetic diameter and its distribution is a measure of the strength of the magnetic dipole within each nanoparticle. The volume median magnetic diameter $\left(D_{\mathrm{m}}\right)$ and geometric deviation $\left(\ln \sigma_{\mathrm{g}}\right)$ can be found by fitting the superparamagnetic equilibrium magnetization curve to the Langevin function,

\footnotetext{
${ }^{8}$. Typically, to demonstrate superparamagnetic behavior in a sample fixed in a solid matrix the field-dependent magnetization curves are recorded at temperatures above the blocking temperature. At temperatures below the blocking temperature, the magnetization will reveal hysteresis loops and the sample coercivity can be measured.

9. Typically, the magnetization is measured for about 100 magnetic field points, logarithmically spaced. This allows one to acquire sufficient data at all logarithmic decades of magnetization, distributed in the field range. The field range available is dependent on the equipment used and must be enough to saturate the ferrimagnets.
} 
weighed using a lognormal size distribution $n_{\downarrow}\left(D_{\mathrm{m}}\right)$ (see Eq. (5)), as suggested by Chantrel et al. [15, 16].

$$
\begin{gathered}
M(\alpha)=M_{\mathrm{s}} \int_{0}^{\infty} n_{\mathrm{v}}\left(D_{\mathrm{m}}\right) L(\alpha) d D_{\mathrm{m}} \quad(3) \\
L(\alpha)=\operatorname{coth}(\alpha)-\frac{1}{\alpha} ; \text { where } \alpha=\frac{\pi \mu_{0} D_{\mathrm{m}}^{3} M_{\mathrm{d}} H}{6 k_{\mathrm{B}} T} \\
n_{\mathrm{v}}\left(D_{\mathrm{m}}\right)=\frac{1}{\sqrt{2 \pi} D \ln \sigma_{\mathrm{g}}} \exp \left(\frac{\ln \left(D_{\mathrm{m}} / D_{\mathrm{pgv}}\right)}{2\left(\ln ^{2} \sigma_{\mathrm{g}}\right)}\right)
\end{gathered}
$$

In Eq. (4), $a$ is the Langevin parameter (ratio of magnetic to thermal energy), $\mu_{0}$ is the permeability of free space, $k_{\mathrm{B}}$ is Boltzmann's constant, and $T$ is the absolute temperature, and $M_{\mathrm{d}}$ is the domain magnetization $(446000 \mathrm{~A} / \mathrm{m}$ or 86 $\mathrm{Am}^{2} / \mathrm{kg}$ for iron oxide).

5. See Fig. 2a for an example of a MH curve for iron oxide nanoparticles embedded in a PSDVB matrix at a concentration of $0.057 \mathrm{mg}_{\mathrm{Fe}_{3} \mathrm{O}_{4}}$. Using Eq. (3), the volume median magnetic diameter is $D_{\mathrm{m}}=14 \mathrm{~nm}$ with $\ln \sigma_{g}=0.246$ at $300 \mathrm{~K}$. The saturation magnetization is $\sim 83 \mathrm{Am}^{2} / \mathrm{kg}_{\mathrm{Fe}_{3} \mathrm{O}_{4}}$, which is in the range of reported values for magnetite and maghemite $\left(80-100 \mathrm{Am}^{2} / \mathrm{kg}\right)$ [26].

6. For the same sample, the magnetic diameter, calculated using the LangevinChantrel model [15, 16], decreases as the saturation field increases (see Fig. 2b). This is because measurements carried in low field strength would cause only larger particles to respond to the magnetic field and thus narrow size distribution is obtained. A true representation of the magnetic diameter and its distribution can be obtained only if the measurements are run at fields much greater than the fields that saturate the particles, such that even smaller particles respond at large magnetic field strengths.

\subsection{Low Field Magnetization}

The extent of magnetic dipole-dipole interactions in a sample can be parameterized using the so-called interaction temperature parameter $T_{0}$. This parameter can be estimated from low field ( $\pm 10-40$ Oe, depending on the sample) MH curves for the sample, measured at various temperatures in a wide temperature range. The sample needs to be solid or embedded in a matrix to restrict particle rotation during the measurement (see Subheading 3.1 or 3.2). Under such conditions, the MH curves will be linear for superparamagnetic samples and the slope of the curves, which corresponds to the initial susceptibility of the sample, will be 
sensitive to the dipole-dipole interactions. The inverse of the initial susceptibility can then be plotted as a function of temperature and fitted to a Curie-Weiss model to obtain $T_{0}$ [27]. We note that $T_{0}$ can be a function of the state of aggregation of the iron oxide nanoparticles in a sample, or of the extent of dipole-dipole interactions in concentrated samples.

1. Embed the magnetic nanoparticles in a solid matrix as explained in Subheadings 3.1 and 3.2. In this protocol, we use iron oxide nanoparticles in a PSDVB matrix. The reader may adapt this protocol to use a polymer or solid matrix compatible with their nanoparticle system (see Notes 1 and 2).

2. Set the desired sample temperature using the equipment software (see Note 8). We use a Superconducting Quantum Interference Device (SQUID) magnetometer MPMS3 from Quantum Design. The reader is assumed to be familiar with the equipment and relevant software described in Subheading 2.4.

3. Measure the magnetization of the sample as a function of increasing the applied magnetic field. Typically, the magnetization is measured for about ten magnetic field steps, uniformly distributed in the field range. The applied field may range from 0.004 to $-0.004 \mathrm{~T}$ and a field ramp of $0.001 \mathrm{~T} / \mathrm{min}$ is suitable.

4. Determine the initial susceptibility $\chi_{0}$ of the sample at different temperatures by calculating the slope of the $M(H)$ curve. Then plot $1 / \chi_{0}$ versus $T$ and fit to the Curie-Weiss model [28]

$$
\chi_{0}=\frac{A}{\left(T-T_{0}\right)} .
$$

where the data is expected to lie in a straight line whose intercept with the $1 / \chi_{0}$ axis corresponds to the interaction temperature parameter $T_{0}$.

5. Figure 3a shows representative low field magnetization curves for iron oxide nanoparticles in a PSDVB matrix in a temperature range of 4-400 K. Note that for temperatures of $136 \mathrm{~K}$ and higher the low field $\mathrm{MH}$ curves are linear and cross the origin, indicating superpamagnetic behavior. For temperatures below $136 \mathrm{~K}$ the low field MH curves still appear linear but no longer cross the origin, indicating the sample has significant remanence and coercivity and is therefore no longer superparamagnetic.

6. Figure $3 \mathrm{~b}$ shows the linear relation between the inverse of the initial susceptibility and temperature for the sample. Typically, this linear relation only holds for a limited temperature range, which usually starts much higher than the temperature for which the sample becomes superparamagnetic. For the sample in Fig. 3b the range starts at about $\sim 260 \mathrm{~K}$, even though the sample appeared superpamagnetic at a temperature of $136 \mathrm{~K}$ in Fig. 3a. Using the model in Eq. (6), the interaction temperature parameter for this sample was determined to be $T_{0}=43.2 \mathrm{~K}$. 


\subsection{Zero-Field-Cooled/Field-Cooled (ZFC/FC) Magnetization Curves}

Whether a given collection of magnetic nanoparticles displays superparamagnetic or ferro/ ferrimagnetic behavior depends on the temperature at which the measurement is made. This is because superparamagnetism corresponds to a state where the energy barrier to dipole moment rotation in the crystal is much smaller than the thermal energy. As such, the temperature at which a collection of magnetic nanoparticles transitions from ferro/ ferrimagnetic behavior to superparamagnetic behavior is an important property. This can be characterized through temperature-dependent magnetization $M(T)$ measurements. The most common way to do this is through so-called Zero-Field-Cooled and Field-Cooled (ZFC/FC) measurements. In such measurements a sample, usually solid or embedded in a solid matrix, begins at a high temperature and at zero field, such that thermal energy completely eliminates any magnetization in the sample. Then the sample is cooled to a low temperature in zero field. The ZFC portion of the plot is obtained by applying a magnetic field once the sample has equilibrated at the lowest temperature and then by measuring the sample's magnetization with increasing temperature. At the lowest temperature the dipoles in the nanoparticles will have the lowest amount of thermal energy, and as such will only align slightly with the applied field, resulting in a small magnetization value. As the temperature increases, the increasing thermal energy of the magnetic dipoles will free them from their initial states, resulting in increased alignment with the field and increasing sample magnetization. However, beyond a certain temperature, referred to as the blocking temperature, further increasing thermal energy will lead to a decrease in the extent of alignment of the magnetic dipoles with the applied field and hence to a decrease in the sample magnetization. The FC portion of the curve is obtained by then measuring magnetization as the sample is cooled back in the applied field to the initial low temperature. In an ideal sample the ZFC and FC curves will overlap at temperatures above the blocking temperature and diverge at temperature below the blocking temperature. For samples with significant dipole-dipole interactions or broad size distributions there will be a significant temperature range above the blocking temperature where the $\mathrm{ZFC}$ and $\mathrm{FC}$ curves do not overlap. Also, the shape of the FC curve below the blocking temperature can also be indicative of the extent of dipole-dipole interactions in a sample. Finally, the blocking temperature can be analyzed to obtain an estimate of the anisotropy constant of the nanoparticles in the sample, by using equations that consider the Néel and Volger-Fulcher models for the magnetic relaxation time.

1. Embed the magnetic nanoparticles in a solid matrix as explained in Subheadings 3.1 and 3.2. In this protocol, we use iron oxide nanoparticles in a PSDVB matrix. The reader may adapt this protocol to use a polymer or solid matrix compatible with their nanoparticle system (see Notes 1 and 2).

2. Heat the sample to the highest working temperature in the absence of an applied magnetic field (see Note 10). Hold this condition for at least $5 \mathrm{~min}$. We use a SQUID magnetometer MPMS3 from Quantum Design. The reader is assumed to

\footnotetext{
10. The low and high temperatures will depend on the actual sample, i.e., transition temperatures of the sample, and equipment temperature range. Polymer matrices have a higher melting temperature (PSDVB $>400 \mathrm{~K}$ ) than long hydrocarbon chains (docosane $315 \mathrm{~K}$, paraffin $326 \mathrm{~K}$ ). We recommend using the widest possible range for the sample and instrument.
} 
be familiar with the equipment and relevant software described in Subheading 2.4 .

3. Set the sample temperature to the lowest value, depending on the actual sample and instrument limit (see Note 11). For iron oxide nanoparticles, we usually start at $10 \mathrm{~K}$. Use cooling rates of $5-10 \mathrm{~K} / \mathrm{min}$ while cooling down.

4. At the lowest temperature, apply a small magnetic field (10-100 Oe, see Note 12).

5. 5. Measure the magnetization of the sample as the temperature increases from 10 to $400 \mathrm{~K}$. Use a temperature sweep rate of $10 \mathrm{~K} / \mathrm{min}$ and measure the magnetic moment at least every $2 \mathrm{~K}$ (see Note 13 ).

6. Decrease the temperature from $400 \mathrm{~K}$ to $10 \mathrm{~K}$ under a small magnetic field (10$100 \mathrm{Oe}$ ) and measure the magnetization at least every $2 \mathrm{~K}$. We recommend using a temperature sweep rate of $10 \mathrm{~K} / \mathrm{min}$ (see Note 13 ).

7. The temperature at which the $\mathrm{ZFC}$ magnetization curve exhibits a maximum is called the blocking temperature $\left(T_{\mathrm{B}}\right)$ (see Fig. 4). Many authors assume that at the blocking temperature the time scale of the measurements is comparable to the Néel relaxation time, given by Eq. (2) and therefore the anisotropy constant is determined by

$$
K=\frac{k_{\mathrm{B}}\left(T_{\mathrm{B}}\right)}{V_{\mathrm{m}}} \ln \left(\frac{\tau_{\mathrm{obs}}}{\tau_{0}}\right)
$$

where $V_{\mathrm{m}}$ is the magnetic volume, calculated using the magnetic diameter determined in Subheading 3.5, $\tau_{\mathrm{obs}}$ is the observation time in seconds, $T_{\mathrm{B}}$ is the blocking temperature, $\tau_{0}$ is the characteristic time, typically assumed to be $\sim 10^{-9} \mathrm{~s}$. The observation time used to calculate anisotropy constant is the ratio between blocking temperature and rate set to reach the temperature, i.e., the sweep rate during measurement $(10 \mathrm{~K} / \mathrm{min}$ for the procedure described above).

8. Recognizing the influence of magnetic dipole-dipole interactions in the relaxation time of the nanoparticles, shtrikman and wolhlfarth [27] proposed the Vogel-Fulcher law, which can be used to estimate the anisotropy constant in samples with significant interactions

\footnotetext{
11. We recommend working with the lowest temperature first and increasing the temperature in $30 \mathrm{~K}$ increments. The temperature range of the experiment depends on the transition temperatures of the sample and equipment temperature range.

12. The blocking temperature becomes a function of the magnitude of this field. At very low field values the blocking temperature is constant, but above 100 Oe it certainly decreases with increasing applied field [39]. The analysis for the anisotropy constant is only valid for the range in which the blocking temperature does not vary with field strength.

13. For better resolution, we recommend measuring the magnetic moment continuously as the field is increased. Also, it is recommended to use small temperature increments to increase the analysis resolution. The blocking temperature will also be a function of the sweep rate for the temperature. This is evident from Eq. (7) if one realizes that the anisotropy constant does not vary, but the observation time changes with temperature sweep rate [29].
} 


$$
K=\frac{k_{\mathrm{B}}\left(T_{\mathrm{B}}-T_{0}\right)}{V_{\mathrm{m}}} \ln \left(\frac{\tau_{\mathrm{obs}}}{\tau_{0}}\right)
$$

where $T_{0}$ is the interaction temperature parameter. To estimate the anisotropy constant, one requires independent knowledge of $T_{0}, \tau_{0}$, and $\tau_{o b s}$.

9. Polydispersity of the sample may be accounted for in Eqs. (7) and (8) by calculating $K$ according to

$$
K=\frac{k_{\mathrm{B}}\left(T_{\mathrm{B}}-T_{0}\right)}{V_{\mathrm{m}}} \ln \left(\frac{\tau_{\mathrm{obs}}}{\tau_{0}}\right) \frac{1}{\exp \left(9 / 2 \ln ^{2} \sigma_{\mathrm{g}}\right)}
$$

10. Figure 4 shows a representative ZFC-FC magnetization curve for the same sample used in Subheading 3.5. The blocking temperature was $T_{\mathrm{B}}=137 \mathrm{~K}$, which is similar to the temperature for which the low field $\mathrm{MH}$ curves became linear and crossed the origin in Fig. 3a. Furthermore, note that the ZFC and FC curves do not overlap until temperatures above $\sim 260 \mathrm{~K}$. This is evidence of significant dipole-dipole interactions in the sample and also explains in part why the linear relationship between inverse initial susceptibility and temperature was observed at temperatures above $\sim 260 \mathrm{~K}$ in Fig. $3 \mathrm{~b}$.

The calculated values of the anisotropy constant $K$ using the maximum temperature of the ZFC curve, and the Néel or Vogel-Fulcher models for the relaxation time are summarized in Table 1. Because the Vogel-Fulcher model takes into account the particle-particle interaction parameter $T_{0}$, the value obtained using the Néel model is larger than that calculated using the VogelFulcher model. However, both values are slightly larger than the magnetocrystalline anisotropy constant of bulk magnetite $13.5 \mathrm{~kJ} / \mathrm{m}^{3}$ [29]. When the polydispersity of the magnetic diameter is included in the analysis, the calculated values decrease and are comparable to the bulk value.

\subsection{Dynamic Magnetic Susceptibility (DMS) as a Function of Temperature}

The transition from ferro/ferrimagnetic behavior to superparamagnetic behavior can also be determined from measurements of the dynamic magnetization of the nanoparticles in response to an oscillating magnetic field as a function of temperature. The measurements are usually done in solid samples or samples in a solid matrix to inhibit the particle rotation (see Subheadings 3.1 and 3.2).

In these measurements, the so-called complex or dynamic magnetic susceptibility $\hat{\chi}(T)=\chi^{\prime}-i \chi^{\prime \prime}$ of the nanoparticles is determined as a function of temperature for various applied oscillating field frequencies. Here, $\chi^{\prime}$ is referred to as the in-phase susceptibility and $\chi$ "is referred to as the out-of-phase susceptibility. This approach poses several advantages over $\mathrm{ZFC} / \mathrm{FC}$ measurements to determine the anisotropy constant of the 
nanoparticles. First, the measurements are done in the absence of an applied constant field and the oscillating excitation field is of small magnitude, such that the Néel and VogelFulcher models for the magnetic relaxation time are expected to be good descriptions of the behavior of the nanoparticles. Second, the observation time is precisely determined by the inverse of the frequency of the applied oscillating magnetic field and can be easily varied by changing the applied oscillating field frequency. This in turn allows one to determine the anisotropy constant without having to assume a value for $\tau_{0}$.

1. Fix the magnetic nanoparticles in a solid matrix as explained in Subheadings 3.1 and 3.2 .

2. Heat the sample to the highest working temperature in the absence of an applied magnetic field (see Note 10). Hold this condition for at least $5 \mathrm{~min}$. We use a SQUID magnetometer MPMS3 from Quantum Design in this protocol. The reader is assumed to be familiar with the equipment and relevant software described in Subheading 2.4.

3. Set the amplitude of the field in the range of $2-5$ Oe.

4. Set the desired frequency of oscillation of the AC field. The same measurement must be repeated at multiple frequencies. The selected frequencies should be in a range that spans several orders of magnitude $(0.1-1000 \mathrm{~Hz})$.

5. Measure the in-phase and out-of-phase components of the magnetic susceptibility of the sample under a constant amplitude oscillating field as the temperature is decreased from 400 to $4 \mathrm{~K}$. During the measurement, start with the highest temperature and decrease temperature at a rate of $6 \mathrm{~K} / \mathrm{min}$ (see Note 14).

6. The in-phase susceptibility $\chi^{\prime}(T)$ curves will display a peak at a temperature that decreases as the applied excitation field frequency decreases. It is assumed that at this peak of the $\chi^{\prime}(T)$ curve the condition $\Omega \tau=1$ applies. To use the Vogel-Fulcher model to interpret the temperature dependence of DMS data [30, 31 , plot $\ln (1 / \Omega)$ versus $1 / T$ and compare to the equation

$$
\ln \frac{1}{\Omega}=\ln \tau_{0}+\left(\frac{K V_{\mathrm{m}}}{k_{\mathrm{B}}\left(T-T_{0}\right)}\right) .
$$

$V_{\mathrm{m}}$ is the magnetic volume. The graph should be linear, with the slope providing an estimate of $K V_{\mathrm{m}}$ and the infinite temperature intercept being a measure of $\tau_{0}$ (which should be in the range of $10^{-9}-10^{-14} s$ [32], otherwise indicating significant interactions and casting doubt on the accuracy of the value of $K$ ).

\footnotetext{
14.We prefer to sweep temperature at fixed frequency, warming the sample after each sweep before starting a new sweep for another frequency. We find this mode of measurement is fast using a Quantum Design MPMS-3, because the instrument can measure the ac susceptibility while it sweeps the field. However, one could also step temperature and measure each frequency at fixed temperature during the cool-down step. We find this mode of measurement to be more effective on a Quantum Design MPMS-XL.
} 
7. To account for sample polydispersity, the expression in Eq. (10) is multiplied by the geometric deviation $\ln \sigma_{\mathrm{g}}$

$$
\ln \frac{1}{\Omega}=\ln \tau_{0}+\left(\frac{K V_{\mathrm{m}}}{k_{\mathrm{B}}\left(T-T_{0}\right)}\right) \exp \left(\frac{9}{2} \ln \sigma_{\mathrm{g}}^{2}\right)
$$

In these equations, $T_{0}$ can be determined using the methods described in Subheading 3.6, or can be assumed to be $T_{0}=0 \mathrm{~K}$ in the case of fitting to the Néel model.

8. Figure 5a shows an example of the $\chi^{\prime}(T)$ curve and Fig. 5 b shows the corresponding plot of $\ln (1 / \Omega)$ versus $1 /\left(T-T_{0}\right)$ using the interaction temperature parameter determined with the Vogel-Fulcher model explained in Subheading 3.6 and assuming $T_{0}=0 \mathrm{~K}$ for the Néel model. The sample was the same sample used in Subheadings 3.5 and 3.6, iron oxide nanoparticles embedded in a PSDVB matrix.

Table 1 summarizes the values of the anisotropy constant calculated using the temperaturedependent dynamic magnetic susceptibility measurements for magnetic nanoparticles with $D_{\mathrm{m}}=14 \mathrm{~nm}, \ln \sigma_{\mathrm{g}}=0.246$, and $V_{\mathrm{m}}=1370 \mathrm{~nm}^{3}$, calculated in step 5 of Subheading 3.5 and the value of $T_{0}=43.2 \mathrm{~K}$ determined from the low field $\mathrm{MH}$ measurements described in step $\mathbf{6}$ of Subheading 3.6. The calculated values are larger than the magnetocrystalline anisotropy constant of bulk magnetite $13.5 \mathrm{~kJ} / \mathrm{m}^{3}$ [29], but decrease when the polydispersity of the magnetic diameter and the interaction temperature parameter are included in the analysis.

\subsection{Dynamic Magnetic Susceptibility as a Function of Frequency}

Dynamic magnetic susceptibility measurements can also be used to determine the mechanism of magnetic relaxation (Néel or Brownian) of a collection of nanoparticles, to estimate the hydrodynamic diameter distribution of particles with predominant Brownian relaxation suspended in a medium of known viscosity, and to determine the viscosity of a liquid with nanoparticles of known hydrodynamic diameter distribution. In these measurements, the dynamic magnetic susceptibility of a sample is measured in a small amplitude oscillating magnetic field at constant temperature and as a function of the frequency of the oscillating magnetic field. Ideally, the amplitude of the oscillating magnetic field remains constant in the whole frequency range of the measurement. However, if the amplitude of the oscillating magnetic field is small enough that the dynamic response is linear with the field amplitude, the measurement can still be completed even if the amplitude of the oscillating field decreases with frequency. Analysis of the frequency dependence of the dynamic magnetic susceptibility can be made for nanoparticles in suspension, for nanoparticles at various temperatures, for nanoparticles suspended in liquids of different viscosities, and for nanoparticles in a solid matrix to obtain information on the mechanism of magnetic relaxation.

1. Suspend the magnetic nanoparticles in a liquid matrix as explained in Subheading 3.3. 
2. Set the desired sample temperature (see Note 15).

3. Set the amplitude of the oscillating magnetic field at a value in the range of 2-5 Oe.

4. Measure the dynamic magnetic susceptibility of the sample as a function of decreasing frequency of oscillation (see Note 16 and 17).

5. Use the Debye model to interpret the measurements of DMS as a function of frequency and obtain information of the hydrodynamic diameter of nanoparticles with predominant Brownian relaxation mechanism. According to the model, when an alternating magnetic field of frequency $\Omega$ is applied to the sample, the in-phase and out-of-phase components of the dynamic magnetic susceptibility are given by

$$
\chi^{\prime}=\chi_{\infty}+\frac{\chi_{0}-\chi_{\infty}}{1+\Omega^{2} \tau^{2}} ; \chi^{\prime \prime}=\frac{\left(\chi_{0}-\chi_{\infty}\right) \Omega \tau}{1+\Omega^{2} \tau^{2}}
$$

where $\chi_{0}$ is the low frequency susceptibility, $\chi_{\infty}$ is the high frequency susceptibility, and $\tau$ is the relaxation time. From Eq. (12), the real component decreases as the frequency increases, whereas the imaginary component has a maximum at $\Omega_{\text {peak }} \tau=1$.

6. To account for polydispersity of nanoparticle hydrodynamic diameters, the susceptibility $\chi^{\prime \prime}$ in Eq. (12) can be weighed using a lognormal size distribution $n_{\mathrm{V}}(D)$.

$$
\begin{aligned}
\chi^{\prime} & =\int_{D_{\mathrm{h}, 0}}^{D_{\mathrm{h}, \infty}} n_{\mathrm{v}}\left(D_{\mathrm{h}}\right) \times\left[\chi_{\infty}+\frac{\chi_{0}-\chi_{\infty}}{1+\Omega^{2} \tau^{2}\left(D_{\mathrm{h}}\right)}\right] \mathrm{d} D_{\mathrm{h}} \\
\chi^{\prime \prime} & =\int_{D_{\mathrm{h}, 0}}^{D_{\mathrm{h}, \infty}} n_{\mathrm{v}}\left(D_{\mathrm{h}}\right) \times\left[\frac{\left(\chi_{0}-\chi_{\infty}\right) \times \Omega \tau\left(D_{\mathrm{h}}\right)}{1+\Omega^{2} \tau^{2}\left(D_{\mathrm{h}}\right)}\right] \mathrm{d} D_{\mathrm{h}}
\end{aligned}
$$

\footnotetext{
15. Since the frequency of the Brownian peak depends on the viscosity of the carrier fluid, the peak frequency can be shifted to the frequency range of the instrument by changing the temperature of the measurement or the viscosity of the carrier liquid. During calculations use the appropriate sample temperature and solvent viscosity.

16. One can also measure the dynamic magnetic susceptibility of the sample as the frequency of oscillation is increased. Both the methods should yield similar results. It is recommended to use low concentrations of particles in solution since the relaxation times given in Eqs. (1) and (2) only apply for infinitely dilute systems with no particle-particle interactions.

17. The oscillation frequency of some commercially available instruments is in the range of a few $\mathrm{Hz}$ to $\mathrm{kHz}$ to $100 \mathrm{~s} \mathrm{of} \mathrm{kHz}$, corresponding to over four orders of magnitude in range. For better resolution measure the DMS for at least ten frequencies per decade, using logarithmically spaced oscillation frequencies.
} 


$$
n_{\mathrm{v}}\left(D_{\mathrm{h}}\right)=\frac{1}{\sqrt{2 \pi} D_{\mathrm{h}} \ln \sigma_{\mathrm{g}}} \exp \left(\frac{\ln \left(D_{\mathrm{h}} / D_{\mathrm{hgv}}\right)}{2\left(\ln ^{2} \sigma_{\mathrm{g}}\right)}\right)
$$

where $D_{\mathrm{hgv}}$ is the volume weighted hydrodynamic diameter, $\ln \sigma_{\mathrm{g}}$ is the geometric deviation, and $\tau\left(D_{\mathrm{h}}\right)$ is the volume weighted relaxation time corresponding to the volume weighted diameter.

7. Using the relation $\tau=1 / \Omega_{\text {peak }}$, obtain the effective relaxation time for a collection of monodisperse nanoparticles. For particles that relax by the Brownian mechanism, $\tau=\tau_{\mathrm{B}}$, and the hydrodynamic diameter of the nanoparticles can be calculated using the peak frequency and the relation

$$
D_{\mathrm{h}}=\sqrt{\frac{2 k_{\mathrm{B}} T}{\pi \eta \Omega_{\text {peak }}}}
$$

8. Similarly, for nanoparticles with predominant Néel relaxation the peak frequency could in principle be used to determine the magnetic diameter of the nanoparticles or the anisotropy constant. However, in those cases, the peak frequency usually occurs at frequencies that are outside the range of commercially available equipment.

9. To verify if the observed peak corresponds to a Brownian peak, measurements at different temperatures or solvents with different viscosities can be used to observe a shift in the peak frequency due to changes in solvent viscosity, whereas the frequency corresponding to the Néel peak would remain constant [20].

10. Plot the DMS spectra of the magnetic nanoparticle samples. Figure 6 shows examples of DMS spectra for two magnetic nanoparticle samples (cobalt ferrite and iron oxide) in 1-octadecene ( $\eta=0.0412 \mathrm{~Pa} \cdot s)$ at $298 \mathrm{~K}$. The difference in the shape of the curves is attributed to the fact that cobalt ferrite nanoparticles possess a much larger anisotropy constant than the iron oxide nanoparticles, and therefore the cobalt ferrite nanoparticles have predominant Brownian relaxation whereas the iron oxide nanoparticles have predominant Néel relaxation. For the iron oxide nanoparticles the peak frequency corresponding to the inverse of the Néel relaxation time would appear at a frequency that is much higher than the maximum frequency that the instrument can apply.

11. Figure 6a corresponds to the DMS spectra of iron oxide nano particles with $D_{h}=$ $17 \mathrm{~nm}\left(\ln \sigma_{g}=0.065\right)$ and $D_{m}=14 \mathrm{~nm}\left(\ln \sigma_{g}=0.246\right)$. Assuming the anisotropy constant for the iron oxide nanoparticles corresponds to the bulk value $K=$ $13 \mathrm{~kJ} / \mathrm{mol}$ [33], the Néel relaxation time of the particles is estimated to be $\tau_{N} \approx$ $9.4 \times 10^{-8}$ s using Eq. (2), whereas using the hydrodynamic diameter the Brownian relaxation time is estimated to be $\tau_{\mathrm{B}} \approx 7.73 \times 10^{-6} \mathrm{~S}$ using Eq. (1). Since $\tau_{\mathrm{N}} \ll \tau_{\mathrm{B}}$, the nanoparticles respond primarily through the Néel relaxation 
mechanism. The corresponding peak frequencies for these two characteristic relaxation times would be $2.6 \times 10^{4} \mathrm{rad} / \mathrm{s}$ for the Brownian mechanism and $1.7 \times$ $10^{6} \mathrm{rad} / \mathrm{s}$ for the Neel relaxation mechanism. The fact that there is no peak at $2.06 \times 10^{4} \mathrm{rad} / \mathrm{s}$ in Fig. 6a indicates that the Brownian mechanism is not dominant for these nanoparticles. Instead, the Néel relaxation mechanism appears to dominate, but the peak cannot be observed as it is expected to lie over one order of magnitude above the frequency range of the instrument. In fact, it appears that the low-frequency tail of the peak is evident at the highest frequencies in the plot of Fig. 6a, although it is difficult to tell if this is instrument artifact introduced at the extreme frequency range for the instrument.

12. The DMS spectrum of Fig. $6 \mathrm{~b}$ may be interpreted using similar arguments. In this case the nanoparticles are cobalt ferrite, with a bulk anisotropy constant of $\mathrm{K}$ $\sim 180 \mathrm{~kJ} / \mathrm{mol}$ [34]. According to dynamic light scattering measurements of these nanoparticles, the hydrodynamic diameter is $D_{\mathrm{h}}=20 \mathrm{~nm}\left(\ln \sigma_{\mathrm{g}}=0.051\right)$. Using Eqs. (1) and (2), the expected Néel relaxation time would be $\tau_{\mathrm{N}} \approx 10^{39} \mathrm{~s}$, whereas the expected Brownian relaxation time would be $\tau_{\mathrm{b}} \approx 1.26 \times 10^{-5}$ s. Since $\tau_{\mathrm{b}} \ll$ $\tau_{\mathrm{n}}$, the nanoparticles respond primarily through the Brownian relaxation mechanism. The corresponding peak frequencies are $10^{-40} \mathrm{rad} / \mathrm{s}$ for the Néel mechanism and $7.94 \times 10^{3} \mathrm{rad} / \mathrm{s}$ for the Brownian mechanism. The experimental peak frequency Fig. $6 \mathrm{~b}$ is $\Omega_{\text {peak }}=4.78 \times 10^{4} \mathrm{rad} / \mathrm{s}\left(7.61 \times 10^{3} \mathrm{~Hz}\right)$. The close agreement between the experimental and Brownian peak frequencies indicates that this sample responds predominantly through Brownian relaxation.

\section{Acknowledgments}

This work was supported by the US National Science Foundation (CBET-143993) and by the US National Institutes of Health (1R01AR068424-01).

\section{References}

1. Pankhurst QA et al. (2009) Progress in applications of magnetic nanoparticles in biomedicine. J Phys D Appl Phys 42 Pag 224001 (15 pp)

2. Roca AG et al. (2009) Progress in the preparation of magnetic nanoparticles for applications in biomedicine. J Phys D Appl Phys 42 Pag 224002 (11 pp)

3. Alvarez-Berrios MP et al. (2014) Magnetic fluid hyperthermia enhances cytotoxicity of bortezomib in sensitive and resistant cancer cell lines. Int J Nanomedicine 9:145-153

4. Kim DH, Nikles DE, Brazel CS (2010) Synthesis and characterization of multifunctional chitosan$\mathrm{MnFe} 2 \mathrm{O} 4$ nanoparticles for magnetic hyperthermia and drug delivery. Materials 3(7):4051-4065 [PubMed: 28883320]

5. Bonini M, Berti D, Baglioni P (2013) Nanostructures for magnetically triggered release of drugs and biomolecules. Curr Opin Colloid Interface Sci 18(5):459-467

6. Hu SH et al. (2008) Core/single-crystal-shell nanospheres for controlled drug release via a magnetically triggered rupturing mechanism. Adv Mater 20(14):2690-2696 [PubMed: 25213891]

7. Kami D et al. (2011) Application of magnetic nanoparticles to gene delivery. Int J Mol Sci 12 (6): 3705-3722 [PubMed: 21747701]

8. Prijic S et al. (2010) Increased cellular uptake of biocompatible superparamagnetic iron oxide nanoparticles into malignant cells by an external magnetic field. J Membr Biol 236 (1):167-179 [PubMed: 20602230] 
9. Na HB, Song IC, Hyeon T (2009) Inorganic nanoparticles for MRI contrast agents. Adv Mater 21(21):2133-2148

10. Qiao RR, Yang CH, Gao MY (2009) Superparamagnetic iron oxide nanoparticles: from preparations to in vivo MRI applications. J Mater Chem 19(35):6274-6293

11. Dhavalikar R et al. (2015) Ferrohydrodynamic modeling of magnetic nanoparticle harmonic spectra for magnetic particle imaging. J Appl Phys 118(17)

12. Goodwill PW et al. (2012) X-Space MPI: magnetic nanoparticles for safe medical imaging. Adv Mater 24(28):3870-3877 [PubMed: 22988557]

13. Krishnan KM (2010) Biomedical nanomagnetics: a spin through possibilities in imaging, diagnostics, and therapy. IEEE Trans Magnet 46(7):2523-2558

14. Neel L (1953) Thermoremanent magnetization of fine powders. Rev Mod Phys 25 (1):293-296

15. Chantrell RW, Popplewell J, Charles SW (1978) Measurements of particle size distribution parameters in ferrofluids. IEEE Trans Magnet 14(5):975-977

16. Rosensweig RE (2013) Ferrohydrodynamics. Courier Corporation Chapter 2, Pag 33-73

17. Jiles D (2015) Introduction to magnetism and magnetic materials. CRC Press, Boca Raton, FL

18. Fannin PC, Perov PA, Charles SW (1999) Complex susceptibility measurements of magnetic fluids over the frequency range $50 \mathrm{MHz}$ to $18 \mathrm{GHz}$. J Phys D Appl Phys 32 (14):1583-1586

19. Shliomis M, Stepanov V (1994) Relaxation phenomena in condensed matter Advances in chemical physics series. Wiley, New York, p. 1

20. Calero-DdelC VL, Santiago-Quinonez DI, Rinaldi C (2011) Quantitative nanoscale viscosity measurements using magnetic nanoparticles and SQUID AC susceptibility measurements. Soft Matter 7(9):4497-4503

21. Dobrotă C-I, Stancu A (2013) What does a first-order reversal curve diagram really mean? A study case: array of ferromagnetic nanowires. J Appl Phys 113(4):043928

22. Carvallo C, Muxworthy AR, Dunlop DJ (2006) First-order reversal curve (FORC) diagrams of magnetic mixtures: micromagnetic models and measurements. Phys Earth Planet In 154(3-4):308322

23. Bruvera I et al. (2015) Determination of the blocking temperature of magnetic nanoparticles: The good, the bad, and the ugly. J Appl Phys 118(18):184304

24. Fabian K (2003) Some additional parameters to estimate domain state from isothermal magnetization measurements. Earth Planet Sci Lett 213(3-4):337-345

25. Caron L et al. (2009) On the determination of the magnetic entropy change in materials with firstorder transitions. J Magn Magn Mater 321(21):3559-3566

26. Cornell RM, Schwertmann U (2003) The iron oxides: structure, properties, reactions, occurrences and uses. John Wiley, New York

27. Shtrikman S, Wohlfarth EP (1981) The theory of the Vogel-Fulcher law of spin-glasses. Phys Lett A 85(8-9):467-470

28. Bradbury A et al. (1984) Magnetic size determination for interacting fine particle systems. IEEE Trans Magnet 20(5):1846-1848

29. Goya GF et al. (2003) Static and dynamic magnetic properties of spherical magnetite nanoparticles. J Appl Phys 94(5):3520-3528

30. Taketomi S (1998) Spin-glass-like complex susceptibility of frozen magnetic fluids. Phys Rev E 57(3):3073-3087

31. Zhang JL, Boyd C, Luo WL (1996) Two mechanisms and a scaling relation for dynamics in ferrofluids. Phys Rev Lett 77(2):390-393 [PubMed: 10062439]

32. Monson TC et al. (2013) Large enhancements of magnetic anisotropy in oxide-free iron nanoparticles. J Magn Magn Mater 331:156-161

33. del Castillo VLCD, Rinaldi C (2010) Effect of sample concentration on the determination of the anisotropy constant of magnetic nanoparticles. IEEE Trans Magnet 46(3):852-859

34. Tung LD et al. (2003) Magnetic properties of ultrafine cobalt ferrite particles. J Appl Phys 93 (10): 7486-7488

35. ASTM (2000) Standard test method for iron in trace quantities using the 1,10-phenanthroline method. In: ASTM standard E394-09, West Conshohocken, PA 
36. Boutry S et al. (2009) Howto quantify iron in an aqueous or biological matrix: a technical note. Contrast Media Mol Imaging 4(6):299-304 [PubMed: 19998319]

37. Danhier P et al. (2012) Electron paramagnetic resonance as a sensitive tool to assess the iron oxide content in cells for MRI cell labeling studies. Contrast Media Mol Imaging 7(3):302-307 [PubMed: 22539400]

38. Mahdavi M et al. (2013) Synthesis, surface modification and characterisation of biocompatible magnetic iron oxide nanoparticles for biomedical applications. Molecules 18 (7):7533 [PubMed: 23807578]

39. Goya GF and Morales M.(2004) Field dependence of blocking temperature in magnetite nanoparticles. J Metast Nanocryst Mater Trans Tech Publ 

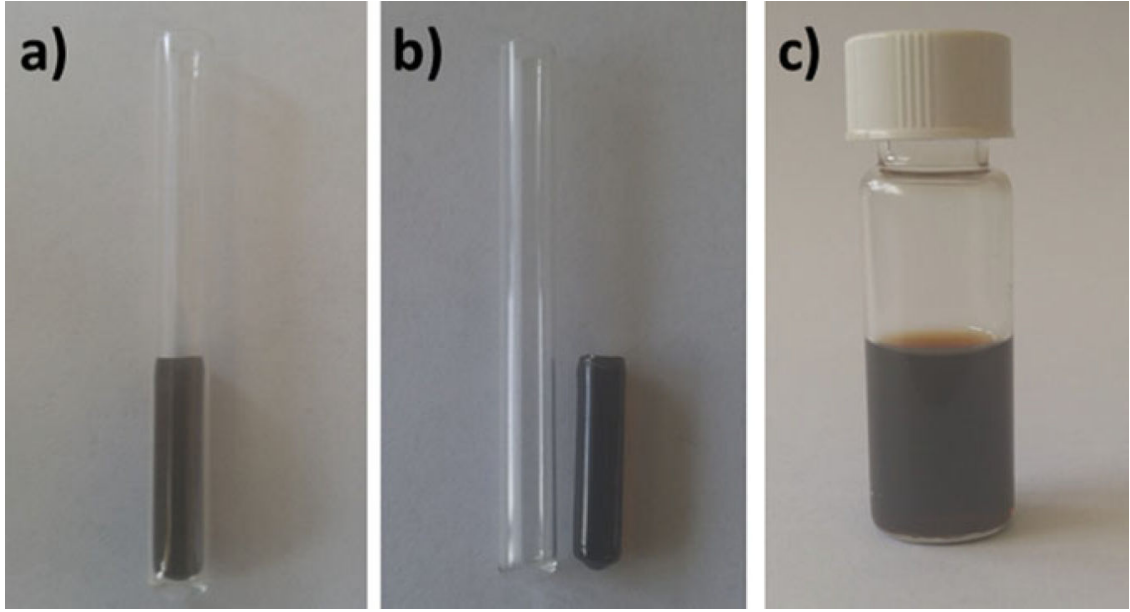

Fig. 1.

Sample preparation. (a) $500 \mu \mathrm{L}$ of the mixture iron oxide nanoparticles and poly(styrenedivinylbenzene) in glass test tubes. (b) Iron oxide nanoparticles immobilized in a poly(styrene-divinylbenzene). (c) Iron oxide nanoparticles in water solution 

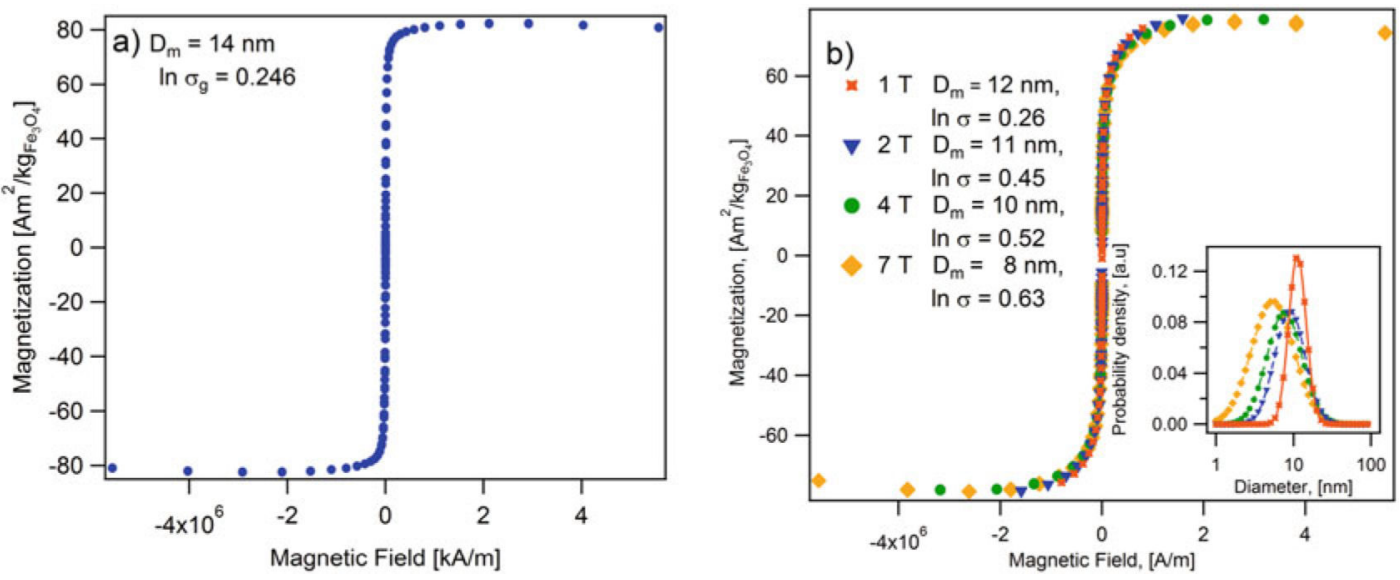

Fig. 2.

(a) Magnetization curves of iron oxide nanoparticles in a poly(styrene-divinylbenzene) matrix measured at different saturation fields. Annotations indicate the magnetic diameter and the geometric deviation calculated using the Langevin-Chantrel model. Inset figure shows the magnetic diameter distribution. (b) Magnetization curve at $300 \mathrm{~K}$ for iron oxide nanoparticles in a poly(styrene-divinylbenzene) matrix. Annotations indicate the magnetic diameter $(14 \mathrm{~nm})$ and the geometric deviation $(\ln \sigma=0.246)$ 

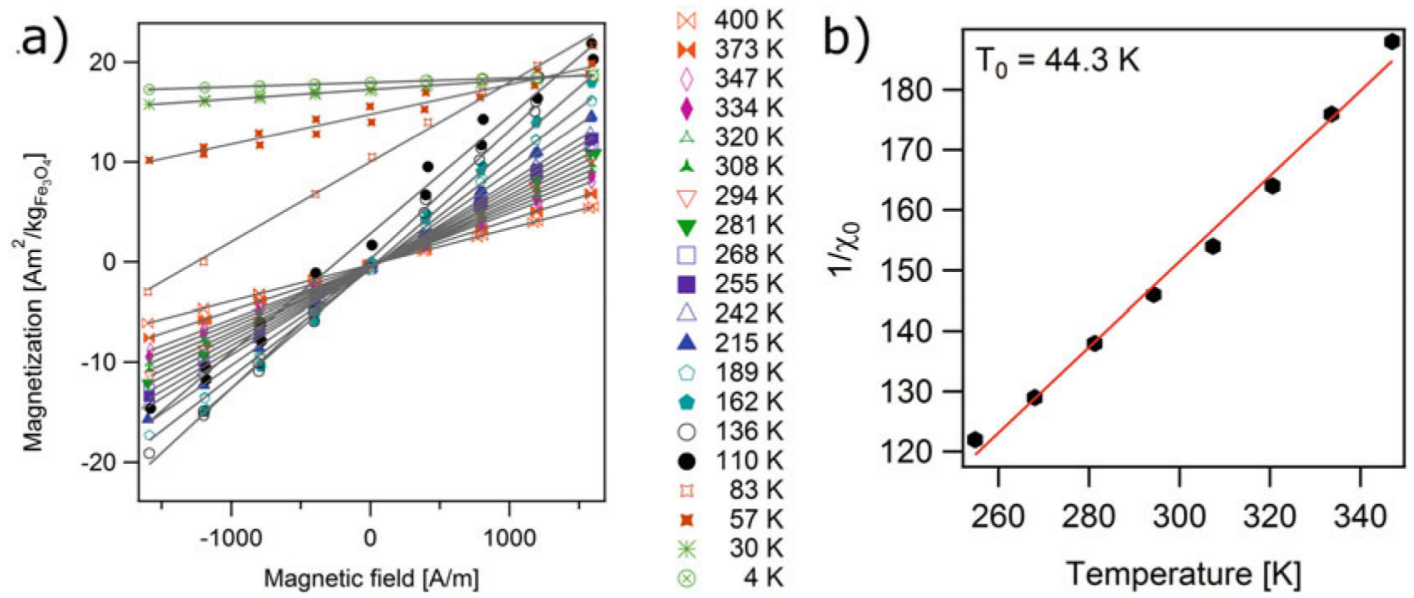

Fig. 3.

(a) Low field magnetization curves for iron oxide nanoparticles in a poly(styrenedivinylbenzene) matrix, at temperatures between $4 \mathrm{~K}$ and $400 \mathrm{~K}$ and (b) initial susceptibility data fitted to Curie-Weiss model 


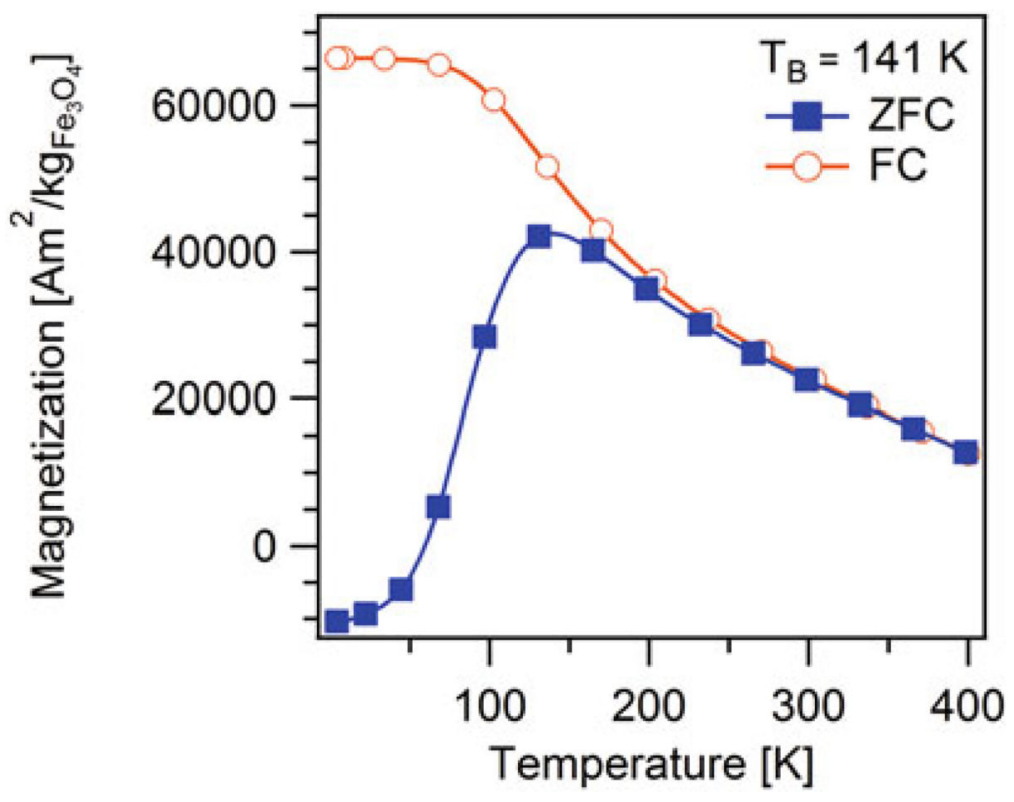

Fig. 4.

Zero field cooled (closed symbols) and field cooled (open symbols) magnetization curve for iron oxide nanoparticles in a poly(styrene-divinylbenzene) matrix, obtained at temperatures between 4 and $400 \mathrm{~K}$ using a $796 \mathrm{~A} / \mathrm{m}$ (10 Oe) field 
a)

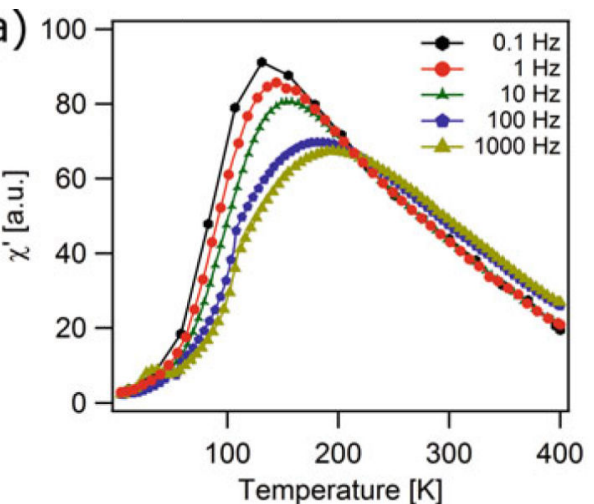

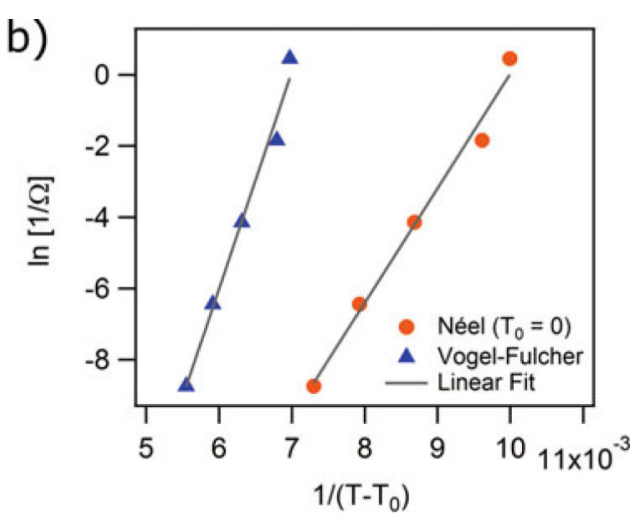

Fig. 5.

(a) In-phase component of the dynamic susceptibility with frequency for iron oxide nanoparticles in a poly(styrene-divinylbenzene) matrix. (b) Inverse applied field frequency as a function of the inverse temperature corresponding to the peak of in-phase component of dynamic susceptibility using the Néel and the Volger-Fuchler model 
a)

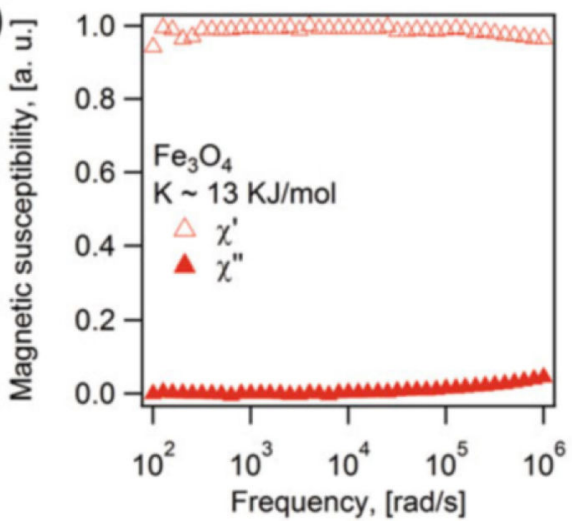

b)

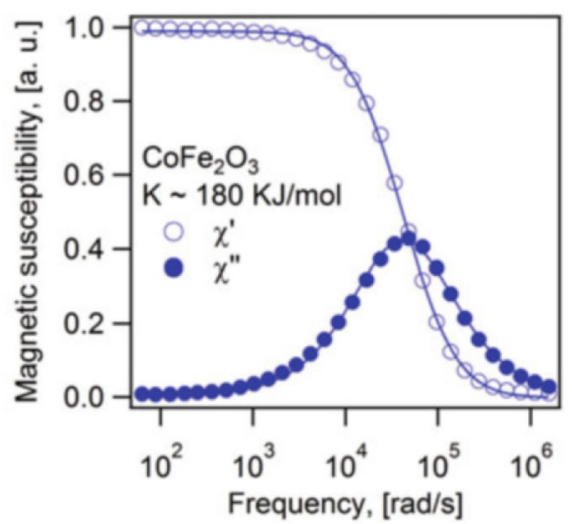

Fig. 6 .

DMS spectra for liquid samples of (a) iron oxide and (b) cobalt ferrite nanoparticles in 1octadecene at $298 \mathrm{~K}$ 


\section{Table 1}

Anistropy constant for Iron Oxide nanoparticles $\left(D_{m}=14 \mathrm{~nm}\right)$ in a PSDVB matrix. Results from ZFC and DMS measurements

\begin{tabular}{llllll}
\hline & & Néel model & \multicolumn{2}{l}{ Vogel-Fulcher model } \\
\cline { 3 - 6 } & & In $\boldsymbol{\sigma}_{\mathbf{g}}=\mathbf{0}$ & In $\boldsymbol{g}_{\mathbf{g}}=\mathbf{0 . 2 4 6}$ & In $\boldsymbol{\sigma}_{\mathbf{g}}=\mathbf{0}$ & In $\boldsymbol{\sigma}_{\mathbf{g}}=\mathbf{0 . 2 4 6}$ \\
\hline ZFC & $K_{\mathrm{ZFC}}\left[\mathrm{KJ} / \mathrm{m}^{3}\right]$ & 46.59 & 35.48 & 27.90 & 21.25 \\
\hline \multirow{2}{*}{ DMS susceptibility } & $\tau_{0}[s]$ & $3.18 \times 10^{-19}$ & $3.18 \times 10^{-19}$ & $1.09 \times 10^{-14}$ & $1.09 \times 10^{-14}$ \\
\hline & $K_{\mathrm{DMS}}\left[\mathrm{KJ} / \mathrm{m}^{3}\right]$ & 61.15 & 46.58 & 32.28 & 24.59 \\
\hline
\end{tabular}

